\title{
Multiple Myeloma Genomics - A Concise Review
}

\author{
Omar Castaneda ${ }^{1}$, Rachid Baz ${ }^{2}$ \\ ${ }^{1}$ Department of Blood \& Marrow \\ Transplant and Cellular Immunotherapy, \\ H. Lee Moffitt Cancer Center and Research \\ Institute, Tampa, FL, USA, ${ }^{2}$ Department \\ of Malignant Hematology, H. Lee Moffitt \\ Cancer Center and Research Institute, \\ Tampa, FL, USA. \\ Correspondence: \\ Rachid.Baz@moffitt.org \\ Tel.: + 18137458212 \\ Fax.: + 18137453071
}

Received: 15 October 2018

Accepted: 25 January 2019

Key Words: Chromosome Aberration - Clonal Evolution - High-Throughput Nucleotide Sequencing.

\begin{abstract}
The aim of this review is to summarize the current knowledge of genomic information in multiple myeloma. Multiple myeloma is a genetically complex plasma cell neoplasm that evolves from pre-malignant stages following genomic evolution leading to the proliferation of malignant plasma cells and the production of monoclonal immunoglobulin. The outcomes of patients with myeloma have dramatically improved over the past decade with the introduction of novel agents. Nevertheless, the disease is considered incurable and displays considerable heterogeneity in clinical presentation, course and survival. This heterogeneity can often be traced to cytogenetic abnormalities in the malignant clone. Accordingly, a large body of literature has examined the impact of genomics on myeloma and risk stratification based on cytogenetics has been adopted. In this review, we will focus on the cytogenetics of multiple myeloma and the prognostic significance as well as possible predictive implications. We will briefly review the existing methodologies relevant to myeloma but explore in greater depth the more novel molecular tools as applied to this disease. Conclusion. The field of genomics in multiple myeloma is rapidly evolving however more translational research is needed to accurately use genomic data as a tool of precision medicine.
\end{abstract}

\section{Introduction}

Multiple myeloma (MM) is a clonal malignancy of terminally differentiated plasma cell representing the second most common hematological malignancy ( $10 \%$ of all hematological malignancies) after non-Hodgkin lymphoma with a globally marked increase in incident cases over that past 25 years ( 1 , 2). This genetically complex disease develops in a multistep process that evolves from premalignant disease states such as monoclonal gammopathy of undetermined significance (MGUS) and smoldering multiple myeloma (SMM) following primary genetic events including chromosomal translocations involving the immunoglobulin heavy-chain genes
(IGH) and aneuploidy. Subsequently, secondary genetics events including copy number abnormalities, DNA hypomethylation and acquired mutations lead to tumor progression $(3,4)$. Genetic events detected at the MGUS stage are considered primary events involved in tumor development and events present at the MM stages that were absent in MGUS are thought to be secondary events leading to tumor progression $(4,5)$.

Since the early 2000s we have observed an accelerated growth of knowledge pertaining to genomic and molecular characterization of MM evolving from metaphase karyotyping and Fluorescent In Situ Hybridization (FISH) to more high-throughput technologies such as gene expression profil- 
ing (GEP) and next generation sequencing The advances in genomic techniques has led to a better appreciation of the underlying genetic abnormalities of multiple myeloma not only at the chromosomal level but at the single gene level showing that multiple myeloma is not a single disease but a collection of diseases with a common clinical phenotype (3). Moreover, myeloma displays significant clonal heterogeneity which is also characterized by different clones having different genomic abnormalities which can impact presentation and drug sensitivity (6). The myeloma plasma cells for the most part reside in the bone marrow but they can also be seen in the peripheral blood and other extramedullary sites. However, it is noteworthy that most of the genomic work has focused on the bone marrow compartment although some investigators have examined the biology of extramedullary disease (7) as well as the genomic characterization of the disease by the means of circulating tumor DNA analysis aiming to accommodate the clonal heterogeneity and multifocal nature of the disease (spatial heterogeneity) however most of this studies are limited by small sample sizes and the lack of consensus about a platform capable of identifying existing and new subclones $(8,9)$. Accordingly, this complex genomic landscape is not yet fully elucidated.

The focus of this review is to summarize the current knowledge of genomic information in MM. For the sake of brevity, we will not focus on already establish methodologies such as metaphase cytogenetics and FISH but rather explore emerging data with new molecular techniques. In addition, we will not review the genomics of other plasma cell dyscrasias including monoclonal gammopathy of undetermined significance, amyloidosis, osteosclerotic myeloma or plasma cell leukemia.

\section{Genetic Alterations}

Using metaphase cytogenetics and FISH, the primary genetic abnormalities in $\mathrm{MM}$ include translocations and trisomies commonly involving odd-numbered chromosomes which are each noted in about $40 \%$ of patients with some overlap $(10,11)$. The primary translocations $(>90 \%)$ in MM usually involve the immunoglobulin heavy chain $(\mathrm{IgH})$ gene locus on chromosome 14 (14q32.33) and one of several partner chromosomes including chromosomes $4,6,11$, 14 , and 20 (Table 1). Less common chromosomes partners include chromosome 12 and 8. Primary trisomies typically involve the odd-numbered chromosomes 3, 5, 7, 9, 11, 15, 19 and/or 21 leading to a hyperdiploid karyotype $(4,10-13)$. In one series, harboring of trisomy 3 or trisomy 5 has significantly better overall survival whereas trisomy 21 was associated with worse outcome (14). Secondary genomic events include chromosomal translocations (MYC), copy number variations and single-nucleotide variants. Monosomy of chromosome 13 and del 13q are the most common secondary cytogenetic abnormalities in MM being detected in $35-40 \%$ and $6-10 \%$ of patients respectively $(12,15)$. Early reports suggested that monosomy 13 or deletion $13 \mathrm{q}$ was associated with worse outcome. However, more recent data in patients treated with bortezomib and/or lenalidomide, the impact of this abnormality is no longer prognostic (16) and it appears that this historical link between del $13 \mathrm{q}$ is a surrogate of its association with high risk cytogenetics lesions. Other abnormalities commonly observed in MM include del $1 \mathrm{p}$, gain 1q, del 17p, and monosomy $17(3,10)$ (Table 1).

The presence of $t(4 ; 14)$ observed in $~ 15 \%$ of patients with MM results in deregulation of the expression of fibroblast growth factor (FGFR3) and multiple myeloma SET (MMSET) and is associated with adverse 
Table 1. Genomic Alterations in Multiple Myeloma*

\begin{tabular}{|c|c|c|c|}
\hline Genomic Event & Genes Involved & Frequency in $\mathrm{MM}^{+}(\%)$ & Prognostic Value \\
\hline \multicolumn{4}{|l|}{ Primary Abnormalities } \\
\hline Trisomies & $\begin{array}{l}\text { Odd-numbered chromosomes: } 3,5,7,9,11 \text {, } \\
15,19 \text { or } 21\end{array}$ & $\sim 45$ & Favorable ${ }^{\ddagger}$ \\
\hline \multirow[t]{5}{*}{ Translocations } & $\mathrm{t}(11 ; 14):$ CCND1 & 15 & Neutral \\
\hline & $\mathrm{t}(4 ; 14): \mathrm{FGR} 3 / \mathrm{MMSET}$ & 15 & Adverse \\
\hline & $t(6 ; 14):$ CCND3 & 2 & Neutral \\
\hline & $t(14 ; 16): M A F$ & 5 & Adverse \\
\hline & $t(14 ; 20):$ MAFB & 1 & Adverse \\
\hline \multicolumn{4}{|c|}{ Secondary Abnormalities } \\
\hline \multirow[t]{3}{*}{ Chromosome gains } & 1q: MCL1, CKS1B, ANP32E or BCL9 & 40 & Adverse \\
\hline & 8q: MYC & 15 & Neutral \\
\hline & 11q: CCND1 & 15 & Neutral \\
\hline \multirow[t]{6}{*}{ Chromosome losses } & 1p: CDKN2C or FAM46C & 30 & Adverse \\
\hline & 12p: CD27 & 15 & Adverse \\
\hline & 14q: TRAF3 & 10 & Not determined \\
\hline & 16q: CYDL or WWOX & 30 & Neutral \\
\hline & 17p:TP53 & 10 & Adverse \\
\hline & 13q: RB1, DIS3, mir15a or mir 16.1 & 40 & Neutral \\
\hline Translocations & Affecting MYC: $t(8 ; 14), t(8 ; 11)$ & 15 & Adverse \\
\hline
\end{tabular}

*Adapted from Manier S, et al. and Kumar SK $(4,12) ;{ }^{\dagger}$ Multiple myeloma; ${ }^{\dagger}$ Trisomy 21 may be associated with worse outcome.

prognosis with poor PFS and OS in different clinical settings $(4,17,18)$. It's worth to mention that despite the poor prognosis associated with $\mathrm{t}(4 ; 14)$ it appears that early treatment of such patients with a proteasome inhibitor may result in survival improvement (19). In terms of prognosis, the most important chromosome arm alteration given its associated aggressive clinical course, poor overall survival and development of extramedullary disease is the monoallelic deletion of $17 \mathrm{p} 13$ (locus of tumor suppressor gene $p 53)(20,21)$. Additional chromosomal changes modulate the outcome of patients with $t(4 ; 14)$ and del(17p) which accounts for the degree of heterogeneity observed in the survival of these high risk patients. OS is impacted in patients harboring $\mathrm{t}(4 ; 14)$ when associated with $\operatorname{del}(13 \mathrm{q} 14), \operatorname{del}(1 \mathrm{p} 32)$ and chromosomal structural changes (>30). Del(1p32) has been also associated with worsening prognosis in patient with del(17p) (22). Most recently Walker et al (23) in a genome-wide analysis of the largest set (1273 NDMM patients) of molecular and clinical data established to date from NDMM, as part of the Myeloma Genome Project, have identified genetic drivers that adversely impact prognosis. Multivariate analysis identified biallelic inactivation of TP53 and gain or amplification of $1 \mathrm{q}$ as being associated with poor PFS (15.4 months) and OS (20.7 months).

Importantly, genetic alterations are further modulated by clinical parameters such as the international staging system (ISS) and serum lactate dehydrogenase (LDH) to impact prognosis. Accordingly, patients with $\mathrm{t}(4 ; 14)$ and ISS1 and normal LDH are expected to fare better than patients with the $t(4 ; 14)$ and ISS3 for example. This is the basis of the revised ISS (17). Based on the revised 
ISS, cytogenetic abnormalities considered to be associated with high risk disease include deletion $17 \mathrm{p}, \mathrm{t}(4 ; 14)$ and $\mathrm{t}(14 ; 16)$.

While there is overall agreement about the prognostic impact of cytogenetic abnormalities in myeloma used to define high risk, it remains unclear whether these abnormalities represent predictive biomarkers. Of the abnormalities detected by FISH, only $\mathrm{t}(11 ; 14)$ has the potential to be used as a predictive marker. As such, myeloma patients harboring the $t(11 ; 14)$ have a single agent response rate of nearly $40 \%$ with the bcl2 inhibitor venetoclax, whereas patients without this translocations are unlikely to respond (24-26).

\section{Gene Expression Profiling}

High-throughput genomic tools such as gene expression profiling (GEP) have been extensively investigated with the goal of predicting patient's outcomes. An initial attempt of molecular classification of MM using GEP identified 5 recurrent translocations, specific trisomies, and expression of cyclin D genes conforming 8 subgroups (11q13, 6p21, 4p16, MAF, D1, D1+D2, and D2) based on cyclin D gene expression and various $14 \mathrm{q} 32$ recurrent translocations (27). On the basis of gene expression profiling studies, several subgroups of multiple myeloma have been identified, which further reflects the genetic heterogeneity of the disease.

In the past years, plasma cell gene-expression signatures designed to specifically identify patients with poor outcome have been developed by several groups including the University of Arkansas for Medical Science (UAMS), Intergroupe Francophone du Myélome (IFM), Skyline 92-HOVON and others (28-30). The UAMS group identified a 70-gene signature by GEP to molecularly define high-risk disease under the treatment platform of Total Therapy 2 (TT2) suggesting that altered transcriptional regulation of genes (nearly half of which map to chromosome 1) may contribute to disease progression. Subsequent multivariate analysis revealed that a 17 -gene subset could predict outcomes as well as the 70-gene model (28). Logistic regression analysis of the 70 -gene score in relation to event free and overall survival data from UAMS TT2/3 series was performed and published in 2016 showing that the 70-gene prognostic risk score is continuously associated with increased risk of 5-year relapse and death (31). Importantly, these GEP signatures were externally validated in datasets including patients treated on various clinical trials and showing continued prognostic significance (32).

An attempt to combine biological and clinical parameters as a prognostic tool was published by Kuiper R, et al (33). Using clinical data of 4750 patients (from the HO65/ GMMG-HD4, UAMS-TT2, UAMS-TT3, MRC-IX, assessment of proteasome inhibition for extending remissions (APEX), and IFM trials) the value of 20 existing risk markers was evaluated. Other than FISH and ISS, gene expression classifiers were used (EMC92, UAMS17, UAMS70, UAMS80, IFM15, MRC-IX6, HM19 and GPI50) showing that combining GEP and ISS data is useful to identify low and high risk MM. Overall while several GEP-based signatures have been developed, there is no consensus on which platform and signature is best and most clinical risk stratification still relies on standard karyotypic analysis and FISH (32). The UAMS has also developed an 80 gene signature (GEP80) by performing GEP analysis in a training set of 142 UAMS-TT3A patients which was subsequently validated in $128 \mathrm{pa}-$ tients in the UAMS-TT3B. The GEP80 signature showed insights into novel mechanisms of resistance to bortezomib with the potential of helping predict response to the agent (34). In addition, while those GEP signature may be prognostic it is unclear if they are predictive and affect the choice of therapy. 


\section{Molecular Information from DNA Sequencing}

Next-generation sequencing has shown a lack of a universal driver mutation in multiple myeloma, and the presence of coexistent subclones of malignant plasma cells with some degree of overlap (3). Investi- gators have reported on results of various DNA-based high-throughput technologies better known as next generation sequencing (NGS) including whole-genome sequencing (WGS) and whole-exome sequencing (WES) to distinguish polymorphisms and characterize the biology of MM. In all studies (summarized in Table 2), a heteroge-

Table 2. Landmark Studies in Multiple Myeloma Genomics

\begin{tabular}{|c|c|c|c|}
\hline Author & Technique(s) & Mutated Gene & Potentially Actionable \\
\hline \multirow[t]{15}{*}{ Walker et al. (19) } & \multirow[t]{15}{*}{ WES ${ }^{*}$} & KRAS & MEK inhibitor \\
\hline & & NRAS & MEK inhibitor \\
\hline & & TP53 & PRIMA-1 analog \\
\hline & & DIS3 & - \\
\hline & & FAM46C & - \\
\hline & & TRAF3 & - \\
\hline & & BRAF & BRAF kinase inhibitor \\
\hline & & $\mathrm{RB} 1$ & - \\
\hline & & CYLD & - \\
\hline & & IRF4 & - \\
\hline & & MAX & - \\
\hline & & HIST1H1E & - \\
\hline & & EGR1 & - \\
\hline & & LTB & - \\
\hline & & FGFR3 & Masitinib \\
\hline \multirow[t]{11}{*}{ Lohr et al. (18) } & WES $^{*}$ & KRAS & MEK inhibitor \\
\hline & \multirow[t]{10}{*}{$\mathrm{WGS}^{+}$} & NRAS & MEK inhibitor \\
\hline & & TP53 & PRIMA-1 analog \\
\hline & & DIS3 & - \\
\hline & & FAM46C & - \\
\hline & & BRAF & BRAF kinase inhibitor \\
\hline & & TRAF3 & - \\
\hline & & $\mathrm{RB} 1$ & - \\
\hline & & CYLD & - \\
\hline & & PRDM1 & - \\
\hline & & ACTG1 & - \\
\hline \multirow[t]{10}{*}{ Chapman et al. (17) } & WES & NRAS & MEK inhibitor \\
\hline & \multirow[t]{9}{*}{$\mathrm{WGS}^{+}$} & KRAS & MEK inhibitor \\
\hline & & FAM46C & - \\
\hline & & DIS3 & - \\
\hline & & TP53 & PRIMA-1 analog \\
\hline & & CCND1 & CDK inhibitor \\
\hline & & PNRC1 & - \\
\hline & & ALOX12B & - \\
\hline & & HLA-A & - \\
\hline & & MAGED1 & - \\
\hline
\end{tabular}


neous mutational landscape was observed and while clonal heterogeneity is an established feature in MM, the subclonal evolution associated with disease progression has not been well explored.

Chapman et al. (35) reported the first results of next generation sequencing in samples of patients with MM. They studied $38 \mathrm{MM}$ patients (WGS in 22 pts, WES in 15 pts and 1 patient sample analyzed by both approaches). This study identified 10 statistically significant protein-coding mutations in MM including NRAS, KRAS, FAM46C, DIS3, TP53, CCND1, PNRC1, ALOX12B, HLA-A and MAGED1 but at a low frequency. One of the thirty eight patients harbored a BRAF kinase mutation leading to the genotyping of an additional $161 \mathrm{MM}$ patients and found BRAF mutations in 7 patients (4\%). The gene set mutation spectrum included genes involved the nuclear factor Kappa B (NF- $\mathrm{B}$ ) pathway, histone methylation, protein translation, and homeostasis.

In 2014, a study by Lohr et al. (36) was designed to address some of the limitations of the Chapman et al study (35) that was only powered to detect commonly mutated genes and didn't examine copy number alterations or clonal heterogeneity due to the small sample size and modest sequence coverage. Parallel sequencing of paired tumor/ normal samples from $203 \mathrm{MM}$ patients showed that eleven genes were recurrently mutated including KRAS, NRAS, TP53, FAM46C, DIS3, BRAF, TRAF3, PRDM1, CYLD, RB1 and ACTG1. Among the 11 significantly mutated genes were five genes (KRAS, NRAS, FAM46C, DIS3 and TP53) previously identified by Chapman et al. (35). The previously tested gene set hypotheses including the mutations of genes in the NF-kB pathway, histone-modifying enzymes and the coagulation cascade retained statistical significance across all 203 patients $(\mathrm{p}<0.05)$ when tested as individual hypotheses.
The largest comprehensive molecular analysis was reported by Walker et al. (20) that performed WES in 463 patients enrolled in a phase III trial (National Cancer Research Institute Myeloma XI Trial) identifying 15 significantly mutated genes: IRF4, KRAS, NRAS, MAX, HIST1H1E, RB1, EGR1, TP53, TRAF3, FAM46C, DIS3, BRAF, LTB, CYLD, and FGFR3. In this cohort the RAS/MAPK pathway was the most frequently mutated pathway (KRAS: $21.2 \%$, NRAS: $19.4 \%$, BRAF: $6.7 \%)$ making up a total of $43.2 \%$. Moreover, mutational activation of the NF-kB pathway genes were identified in $17 \%$ of cases. In this study FGFR3 was found to be mutated solely in the $t(4 ; 14)$ group and the transcriptional regulator EGR1 mutation in the hyperdiploid samples.

Interestingly, the only recurrent mutations that significantly affect survival outcomes are those observed in TP53. The coexistence of del $17 \mathrm{p}$, and TP53 mutations (25-40\% of patients harboring del $17 p)$ appears to cumulatively increase the risk of poor outcomes highlighting the hierarchical interaction between abnormalities of different types $(4,12,21)$. Most chromosome 17 deletions are hemizygous and are observed in around $10 \%$ of patients with newly diagnosed MM however, the frequency increases up to $80 \%$ in advanced disease. The TP53 gene is located within a minimally deleted region on $17 \mathrm{p} 13$ and functions as a transcriptional regulator influencing cellcycle arrest, DNA repair, and apoptosis in response to DNA damage $(4,21,37)$.

These sequencing studies have examined the mutational landscape in MM showing that despite genetic heterogeneity the most frequently mutated genes belong to a limited number of pathways (Table 3) as well as the lack of a universal driver mutation and the presence of coexistent subclones. More importantly, in the current era of personalized medicine when physicians aim to tailor the appropriate therapy to each patient on the 
Table 3. Frequently Mutated Genes in Multiple Myeloma*

\begin{tabular}{lll}
\hline Gene & Frequency (\%) & Function \\
\hline KRAS & $20-25$ & MAPK signaling pathway (cell survival and growth) \\
\hline NRAS & $23-25$ & MAPK signaling pathway (cell survival and growth) \\
\hline TP53 & $8-15$ & Tumor suppressor involved in response to DNA damage and apoptosis \\
\hline DIS3 & 11 & Exosome endoribonuclease \\
\hline FAM46C & $\sim 11$ & Unclear \\
\hline BRAF & $6-15$ & MAPK signaling pathway (cell survival and growth) \\
\hline TRAF3 & $3-6$ & NF-KB signaling pathway (cell survival and proliferation) \\
\hline ROBO1 & $2-5$ & Transmembrane receptor involved cell growth though crosstalk with MET signaling \\
\hline CYLD & $2-3$ & NF-KB signaling pathway (cell survival and proliferation) \\
\hline EGR1 & $4-6$ & Transcription factor \\
\hline SP140 & $5-7$ & Antigen-response mechanisms in mature B cells \\
\hline FAT3 & $4-7$ & Cadherin superfamily member (cell adhesion) \\
\hline CCND1 & 3 & Cell cycle progression \\
\hline
\end{tabular}

*From Kumar SK (12).

basis of genomic data, the identification of driver mutations in MM is promising especially for those patients harboring actionable mutations/pathways. To date however, the only evidence of precision medicine is related to BRAF mutation found in about $4 \%$ of patients and case reports had noted response with the BRAF inhibitor, vemurafenib $(38,39)$.

\section{Clonal Evolution and Subclonality}

Despite being characterized by the secretion of a unique monoclonal protein in the majority of patients, a degree of heterogeneity is observed at the molecular level, which suggests a Darwinian evolution of MM (35, $36,6,40)$. This heterogeneity is observed as soon as the monoclonal gammopathy of undetermined significance stage, meaning that immortalized plasma cells diverge very early in their evolution $(40,41)$. Keats et al. have examined the genomic changes over time in 28 patients with multiple myeloma (42). They noted that about a third of the patients (especially low risk hyperdiploid patients) had stable genome over time. The rest of the patients had clonal changes characterized by either clonal heterogeneity at diagnosis or linear evolution. Of interest, patients with high risk cytogenetics had more genomic changes and only one high risk patient had genomic stability. The same authors studied the clonal dynamics of 1 patient with $t(4 ; 14)$ at 7 time points and noted the clonal dynamics in the face of treatment selection.

Next generation sequencing has shown a lack of universal driver mutation, presence of coexistent subclones and oligoclonality in MM which leads to various type of evolution of the disease over time $(3,40)$. Clonal evolution in multiple myeloma before and after therapy can follow several patterns including branching clonal development, subclonal shift, linear clonal pattern and clonal stability (4). In patients with branching clonal evolution (estimated $30 \%$ of patients), one or more subclones appear at a later time point, whereas others subclones have disappeared. In patients with a subclonal shift, the subclones at diagnosis are also present at relapse, but the frequency of the subclones has changed throughout the disease course and one clone has become more dominant than another. In patients with a linear clonal 


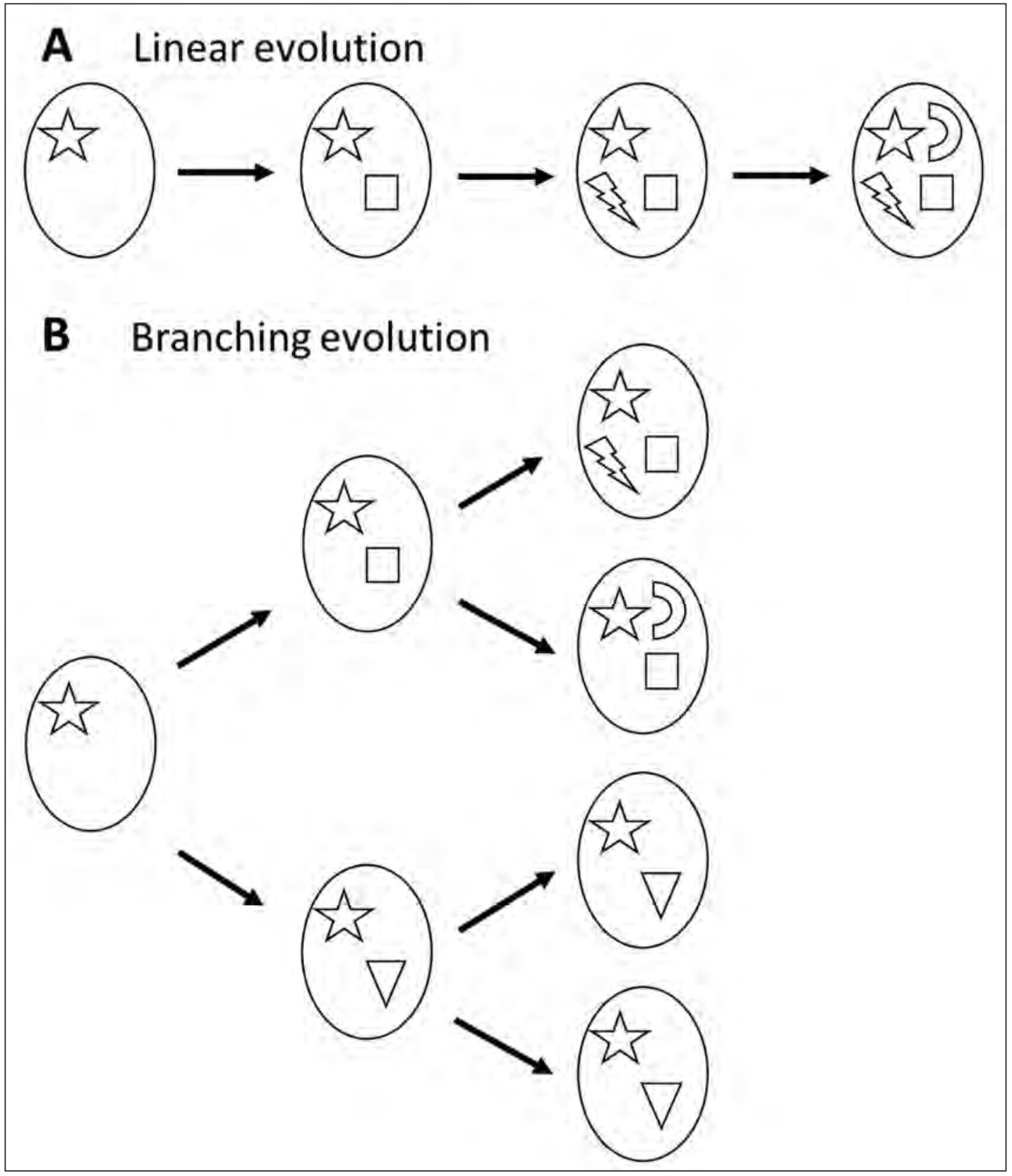

Figure 1. Myeloma is characterized by two types of subclonal evolution (A) a linear one with acquisition of novel mutations over time in the clone and (B) a branching evolution where subclones diverge with subclonal acquisition of novel mutations. From Robiou du Pont S (40).

evolution ( $\sim 40 \%$ of patients), a new subclone has emerged between diagnosis and relapse, which was absent at diagnosis (40). In patients with clonal stability (approximately $30 \%$ of patients) the same composi- tion of clonal and subclonal heterogeneity is found before and after treatment suggesting that all subclones are affected by therapy and will repopulate equivalently (4) (Figure 1). As noted by Keats et al. this may be more 
commonly noted in patients with low risk disease.

The therapeutic implications of clonal heterogeneity and clonal evolution are key areas in multiple myeloma and may have therapeutic implications as previously noted by Fakhri et al. (41) including subclone drug resistant allowing future dominance, targeted therapy of the main clone to maximize effectiveness, combination therapy to overcome branching clonal evolution and therapy recycling based on reemergence of clone(s). Targeted agents will more than likely have to be combined with standardof-care agents, and such regimens might need to be adjusted over time based on the evolving clonal architecture, while maintaining the broadly active backbone combinations (12).

\section{Summary}

The availability of more affordable and high throughput genomic tools has led to an improved understanding of the genomic landscape of multiple myeloma. While the complexity and heterogeneity of the disease continue to make personalized medicine a challenge for myeloma patients, it is our opinion that this genomic revolution will undoubtedly lead to precision medicine in myeloma in the near future. Importantly, in addition to an improved understanding of tumor genomics, an in-depth assessment of the tumor microenvironment (including the immune microenvironment) and the host are needed to more completely characterize the disease, identify new targets and develop better therapies for myeloma patients.

Authors' Contributions: Conception and design: OC and RB; Acquisition, analysis, and interpretation of data: OC and RB; Drafting the article: OC; Revising it critically for important intellectual content: RB; Approved final version of the manuscript: $O C$ and $R B$.

Conflict of Interest: The authors declare that they have no conflict of interest.

\section{References}

1. Siegel RL, Miller KD, Jemal A. Cancer statistics, 2018. CA Cancer J Clin. 2018;68(1):7-30.

2. Cowan AJ, Allen C, Barac A, Basaleem H, Bensenor I, Curado MP, et al. Global Burden of Multiple Myeloma: A Systematic Analysis for the Global Burden of Disease Study 2016. JAMA Oncol. 2018;4(9):1221-7.

3. Kumar SK, Rajkumar SV, Kyle RA, van Duin M, Sonneveld P, Mateos MV, et al. Multiple myeloma. Nat Rev Dis Primers 2017;3:17046.

4. Manier S, Salem KZ, Park J, Landau DA, Getz G, Ghobrial IM. Genomic Complexity of multiple myeloma and its clinical implications. Nat Rev Clin Oncol. 2017;14(2):100-13.

5. Rajkumar SV, Dimopoulos MA, Palumbo A, Blade J, Merlini G, Mateos MV, et al. International Myeloma Working Group updated criteria for the diagnosis of multiple myeloma. Lancet Oncol. 2014;15(12):e538-48.

6. Brioli A, Melchor L, Cavo M, Morgan GJ. The impact of intra-clonal heterogeneity on the treatment of multiple myeloma. Br J Haematol. 2014;165(4):441-54.

7. Usmani SZ, Heuck C, Mitchell A, Szymonifka J, Nair B, Hoering A, et al. Extramedullary disease portends poor prognosis in multiple myeloma and is over-represented in high-risk disease even in the era of novel agents. Haematologica. 2012;97(11):1761-7.

8. Mithraprabhu S, Khong T, Ramachandran M, Chow A, Klarica D, Mai L, et al. Circulating tumour DNA analysis demonstrates spatial mutational heterogeneity that coincides with disease relapse in myeloma. Leukemia. 2017;31(8):1695705.

9. Rasche L, Chavan SS, Stephens OW, Patel PH, Tytarenko R, Ashby C, et al. Spatial genomic heterogeneity in multiple myeloma revealed by multiregion sequencing. Nat Commun. 2017;8(1):268.

10. Morgan GJ, Walker BA, Davies FE. The genetic architecture of multiple myeloma. Nat Rev Cancer. 2012;12(5):335-48.

11. Kumar S, Fonseca R, Ketterling RP, Dispenzieri A, Lacy MQ, Gertz MA, et al. Trisomies in multiple myeloma: impact on survival in patients with high-risk cytogenetics. Blood. 2012;119(9):21005 .

12. Kumar SK, Rajkumar SV. The multiple myelomas current concepts in cytogenetic classification and therapy. Nat Rev Clin Oncol. 2018;15(7):409-21.

13. Debes-Marun CS, Dewald GW, Bryant S, Picken E, Santana-Dávila R, González-Paz N, et al. Chro- 
mosome abnormalities clustering and its implications for pathogenesis and prognosis in myeloma. Leukemia. 2003;17(2):427-36.

14. Chretien ML, Corre J, Lauwers-Cances V, Magrangeas F, Cleynen A, Yon E, et al. Understanding the role of hyperdiploidy in myeloma prognosis: which trisomies really matter? Blood. 2015;126(25):2713-9.

15. Binder M, Rajkumar SV, Ketterling RP, Greipp PT, Dispenzieri A, Lacy MQ, et al. Prognostic implications of abnormalities of chromosome 13 and the presence of multiple cytogenetic high-risk abnormalities in newly diagnosed multiple myeloma. Blood Cancer J. 2017;7(9):e600.

16. Jagannath S, Richardson PG, Sonneveld P, Schuster MW, Irwin D, Stadtmauer EA, et al. Bortezomib appears to overcome the poor prognosis conferred by chromosome 13 deletion in phase 2 and 3 trials. Leukemia. 2007;21(1):151-7.

17. Palumbo A, Avet-Loiseau H, Oliva S, Lokhorst HM, Goldschmidt H, Rosinol L, et al. Revised International Staging System for Multiple Myeloma: A Report From International Myeloma Working Group. J Clin Oncol. 2015;33(26):2863-9.

18. Chang H, Sloan S, Li D, Zhuang L, Yi QL, Chen CI, et al. The $\mathrm{t}(4 ; 14)$ is associated with poor prognosis in myeloma patients undergoing autologous stem cell transplant. Br J Haematol. 2004;125(1):64-8.

19. Avet-Loiseau H, Leleu X, Roussel M, Moreau P, Guerin-Charbonnel C, Caillot D, et al. Bortezomib plus dexamethasone induction improves outcome of patients with $\mathrm{t}(4 ; 14)$ myeloma but not outcome of patients with del(17p). J Clin Oncol. 2010;28(30):4630-4.

20. Walker BA, Boyle EM, Wardell CP, Murison A, Begum DB, Dahir NM, et al. Mutational Spectrum, Copy Number Changes, and Outcome: Results of a Sequencing Study of Patients With Newly Diagnosed Myeloma. J Clin Oncol. 2015;33(33):391120.

21. Lodé L, Eveillard M, Trichet V, Soussi T, Wuillème S, Richebourg S, et al. Mutations in TP53 are exclusively associated with del(17p) in multiple myeloma. Haematologica. 2010;95(11):1973-6.

22. Hebraud B, Magrangeas F, Cleynen A, LauwersCances V, Chretien ML, Hulin C, et al. Role of additional chromosomal changes in the prognostic value of $t(4 ; 14)$ and del $(17 \mathrm{p})$ in multiple myeloma: the IFM experience. Blood. 2015;125(13):2095100 .

23. Walker BA, Mavrommatis K, Wardell CP, Ashby TC, Bauer M, Davies F, et al. A high-risk, DoubleHit, group of newly diagnosed myeloma identified by genomic analysis. Leukemia. 2019;33(1):159170. Epub 2018 Jul 2.

24. Kumar S, Vij R, Kaufman JL, Mikhael J, Facon T, Pegourie B, et al. Venetoclax monotherapy for relapsed/refractory multiple myeloma: safety and efficacy results from a phase I study. Blood. 2016;128(22):488.

25. Kumar S, Kaufman JL, Gasparetto C, Mikhael J, Vij R, Pegourie B, et al. Efficacy of venetoclax as targeted therapy for relapsed/refractory $t(11 ; 14)$ multiple myeloma. Blood. 2017;130(22):2401-9.

26. Moreau P, Chanan-Khan A, Roberts AW, Agarwal AB, Facon T, Kumar S, et al. Promising efficacy and acceptable safety of venetoclax plus bortezomib and dexamethasone in relapsed/refractory MM. Blood. 2017;130(22):2392-400.

27. Bergsagel PL, Kuehl WM, Zhan F, Sawyer J, Barlogie B, Shaughnessy J. Cyclin D dysregulation: an early and unifying pathogenic event in multiple myeloma. Blood. 2005;106(1):296-303.

28. Shaughnessy JD Jr, Zhan F, Burington BE, Huang Y, Colla S, Hanamura I, et al. A validated gene expression model of high-risk multiple myeloma is defined by deregulated expression of genes mapping to chromosome 1. Blood. 2007;109(6):227684.

29. Decaux O, Lodé L, Magrangeas F, Charbonnel C, Gouraud W, Jézéquel P, et al. Prediction of survival in multiple myeloma based on gene expression profiles reveals cell cycle and chromosomal instability signatures in high-risk patients and hyperdiploid signatures in low-risk patients: a study of the Intergroupe Francophone du Myélome. J Clin Oncol. 2008;26(29):4798-805.

30. Kuiper R, Broyl A, de Knegt Y, van Vliet MH, van Beers EH, van der Holt B, et al. A gene expression signature for high-risk multiple myeloma. Leukemia. 2012;26(11):2406-13.

31. Van Laar R, Bender RA, Zielinski A, Leigh Kenton, Barlogie B, Morgan GJ. Use of multiple myeloma 70-gene prognostic risk score as a continuous predictor of patient outcome. Blood. 2016;128(22):5614.

32. Nishihori T, Shain K. Insights on genomic and molecular alterations in multiple myeloma and their incorporation towards risk-adapted treatment strategy: concise clinical review. Int J Genomics. 2017;2017:6934183.

33. Kuiper R, van Duin M, van Vliet $M H$, Broijl A, van der Holt B, El Jarari L, et al. Prediction of high- and low-risk multiple myeloma based on gene expression and the International Staging System. Blood. 2015;126(17):1996-2004. 
34. Shaughnessy JD Jr, Qu P, Usmani S, Heuck CJ, Zhang Q, Zhou Y, et al. Pharmacogenomics of bortezomib test-dosing identifies hyperexpression of proteasome genes, especially PSMD4, as novel high-risk feature in myeloma treated with Total Therapy 3. Blood. 2011;118(13):3512-24.

35. Chapman MA, Lawrence MS, Keats JJ, Cibulskis K, Sougnez C, Schinzel AC, et al. Initial genome sequencing and analysis of multiple myeloma. Nature. 2011;471(7339):467-72.

36. Lohr JG, Stojanov P, Carter SL, Cruz-Gordillo P, Lawrence MS, Auclair D, et al. Widespread genetic heterogeneity in multiple myeloma: implications for targeted therapy. Cancer Cell. 2014;25(1):91101.

37. Chin M, Sive JI, Allen C, Roddie C, Chavda SJ, Smith D, et al. Prevalence and timing if TP53 mutations in del(17p) myeloma and effect on survival. Blood Cancer J. 2017;7(9):e610.
38. Andrulis M, Lehners N, Capper D, Penzel R, Heining C, Huellein J, et al. Targeting the BRAF V600E mutation in multiple myeloma. Cancer Discov. 2013;3(8):862-9.

39. Mey UJM, Renner C, von Moos R. Vemurafenib in combination with cobimetinib in relapsed and refractory extramedullary multiple myeloma harboring the BRAF V600E mutation. Hematol Oncol. 2017;35(4):890-3.

40. Robiou du Pont S, Cleynen A, Fontan C, Attal M, Munshi N, Corre J, et al. Genomics of Multiple Myeloma. J Clin Oncol. 2017;35(9):963-7.

41. Fakhri B, Vij R. Clonal evolution in multiple myeloma. Clin Lymphoma Myeloma Leuk. 2016;16(S1):S130-4.

42. Keats JJ, Chesi M, Egan JB, Garbitt VM, Palmer SE, Braggio E, Vet al. Clonal competition with alternating dominance in multiple myeloma. Blood. 2012;120(5):1067-76. 